\title{
Caractérisation Biochimique De La Pulpe des Fruits Du Prunier Noir (Vitex Doniana) De La Côte d'Ivoire
}

\section{Kone Haffiata Soro, Ing-Doctorante}

Ecole Doctorale Polytechnique de Yamoussoukro (EDP), Laboratoire des

Procédés Industriels de Synthèses de l'Environnement et des Energies

Nouvelles (LAPISEN), Institut National Polytechnique Félix Houphouët Boigny de Yamousoukro, (INP-HB). Côte d'Ivoire Université Péléforo Gon

Coulibaly de Korhogo (UPGC), Côte d'Ivoire

$$
\begin{gathered}
\text { Kone Kisselmina Youssouf, PhD, MA } \\
\text { Akaki Koffi David, PhD, MC } \\
\text { Soro Doudjo, PhD, MA } \\
\text { Elleingang Fattoh Eric, PhD, MC } \\
\text { Assidjo Nogbou Emmanuel, PhD, PT }
\end{gathered}
$$

Département de Formation et de Recherche en Génie Chimique et

Agroalimentaire (DFR-GCAA). Laboratoire des Procédés Industriels de Synthèses de l'Environnement et des Energies Nouvelles (LAPISEN), Institut

National Polytechnique Félix Houphouët Boigny de Yamousoukro,

(INP-HB), Côte d'Ivoire

\section{Doi: 10.19044/esj.2018.v14n3p252 URL:http://dx.doi.org/10.19044/esj.2018.v14n3p252}

\begin{abstract}
Black plum, the fruit of Vitex doniana, was harvested in three (3) regions belonging to different climatic zones in northern Côte d'Ivoire. Biochemical characteristics of the pulps were studied in order to highlight their nutritional potential and the variability of this composition according to study areas. From the conventional biochemical analyzes used, it appears that the fruit pulp is acidic and mostly made up of water. It is rich in sugars and has antioxidant power between $18.63 \pm 0.02$ and $35.54 \pm 0.01 \mu \mathrm{g} \cdot \mathrm{mL}^{-1}$. There is also the presence of polyphenols $(166.67 \pm 0.01-196.67 \pm 0.01 \mathrm{mEq}$ A.G./100g) and vitamin C (14.34 $\pm 0.02-15.05 \pm 0.07 \mathrm{mg} / 100 \mathrm{~g})$. Ashes consist of minerals such as magnesium, potassium and calcium. The analysis of the variance showed that the mean of the parameters studied are significantly different from one region to the other, except the ash rate and vitamin $\mathrm{C}$ content. In view of its nutritional quality, black plum presents itself as an interesting fruit to be taken into account in the prevention of certain dietary imbalances and which can even intervene at low cost in the treatment
\end{abstract}


of certain pathologies.

Keywords: Vitex doniana, black plum, nutritional potential, natural variability, Côte d'Ivoire

\section{Résumé}

La prune noire, fruit du Vitex doniana a été récoltée dans trois (3) régions appartenant à des zones climatiques différentes du Nord de la Côte d'Ivoire. Les caractéristiques biochimiques des pulpes ont fait l'objet d'une étude qui visait la mise en exergue de leur potentiel nutritionnel et la variabilité de cette composition suivant les zones d'étude. Des analyses biochimiques classiques utilisées, il ressort que la pulpe des fruits est acide et majoritairement constituée d'eau. Elle est riche en sucres et a un pouvoir antioxydant compris entre $18,63 \pm 0,02$ et $35,54 \pm 0,01 \mu \mathrm{g} \cdot \mathrm{mL}^{-1}$. On note également la présence de polyphénols $(166,67 \pm 0,01-196,67 \pm 0,01 \mathrm{mEq}$ A.G./100g) et de la vitamine C $(14,34 \pm 0,02-15,05 \pm 0,07 \mathrm{mg} / 100 \mathrm{~g})$. Les cendres se composent de minéraux tels le magnésium, le potassium et le calcium. L'analyse de la variance a montré que les moyennes des paramètres étudiés sont significativement différentes d'une région à l'autre, hormis le taux de cendres et la teneur en vitamine C. Au regard de sa qualité nutritionnelle, la prune noire se présente comme un fruit intéressant à prendre en compte dans la prévention de certains déséquilibres alimentaires et pouvant même intervenir à moindre frais dans le traitement de certaines pathologies.

Mots-clés: Vitex doniana, prune noire, potentiel nutritionnel, variabilité naturelle, Côte d'Ivoire

\section{Introduction}

En Côte d'Ivoire comme dans la plupart des pays pauvres et ceux en voie de développement, la question de la sécurité alimentaire demeure un défi majeur pour lequel le gouvernement affecte parfois de gros budgets (UEMOA/PRSA, 2002 ; MSHP/PNN, 2009 ; Schartz et al., 2010 ; Tapé et al. 2015). Dans le nord du pays, les carences alimentaires atteignent pour certaines zones, des taux record jusqu'à $40 \%$ pour la malnutrition aigüe globale et plus de 5\% pour la malnutrition aigüe sévère (FAO, 2008 ; Schartz et al., 2010 ; N'goran, 2014 ). Les couches sociales les plus touchées sont les femmes en âge de procréer et les enfants (OMS, 2009 ; Stevens et al., 2013 ; N'goran, 2014).

Une possibilité de réduire cette carence nutritionnelle est la valorisation des produits alimentaires locaux et plus précisément les fruits forestiers car leur potentiel nutritif et leur disponibilité quasi gratuite en font des denrées accessibles à toutes les couches sociales. 
La prune noire ou prunier des savanes, très prisée par ces populations (Ambé, 2001), pourrait constituer une source non négligeable d'éléments nutritifs vue l'importance capitale que revêt son plant, le prunier des Savanes (Vitex doniana), dans la satisfaction de nombreux besoins sanitaires locaux.

En effet, le prunier des savanes a un potentiel thérapeutique très important. Toutes les parties de la plante entrent dans la composition de remèdes traditionnels. Les fruits, les feuilles et les racines ont des propriétés antiseptiques et vulnéraires. Les feuilles possèdent des propriétés antimicrobiennes (Nwachukwu et al., 2010), traitent les maux de tête, la rougeole, les éruptions cutanées, la fièvre, la varicelle et l'hémiplégie. Elles servent aussi de tonique, d'antalgique et de fébrifuge, et permettent de soigner des maladies respiratoires, les conjonctivites purulentes et autres affections oculaires (Malzy, 1954 ; Okafor, 1991 ; Arbonnier, 2000 ; Ky et al., 2008 ; Sanogo et al., 2009 ). L'écorce peut être utilisée pour lutter contre les saignements à l'accouchement, les affections du rein et a également un effet hypotenseur et hépatoprotecteur. Le mélange de l'écorce, des feuilles et des racines traite l'ictère, les douleurs abdominales, la lèpre, les diarrhées infantiles (Ky et $a l$, 2008). Les racines soignent les maux de dents. Les ramilles servent de bâtons à mâcher pour curer les dents et protègent les dents contre la carie (Ky et $a l, 2008$ ). En grande partie consommée frais, son fruit possède aussi des vertus médicinales et est utilisé dans le traitement de certains troubles comme la fatigue, la constipation, les carences en vitamine A et B, l'anémie, la jaunisse, la lèpre et la dysenterie (CTA, 2007 ; Ky, 2008 ; Ngwamashi, 2009 ; Dadjo et al., 2012).

Le fruit du Vitex doniana est majoritairement consommé par les populations du Nord de la Côte d'Ivoire, toutefois, il n'existe aucune donnée sur la composition nutritionnelle de ce fruit en Côte d'Ivoire. D'où la motivation de ces recherches qui aboutiront à la mise en exergue de ses atouts.

L'étude a été menée dans trois (3) régions du nord de la Côte d'Ivoire : Bagoué (localité : Boundiali 9³2' Nord ; 6²9' Ouest), Poro (localités : Sirasso 9०7'60' 'Nord ; 6 6'0', Ouest et Nafoun 9'20'544 Nord ; 6²0'5', Ouest) et Tchologo (localité : Ferkessédougou 9³5'37', Nord ; 5'11'50', Ouest). Ces régions présentent des différences tant au niveau du type de végétation qu'au niveau des types de sols (AISA, 1991). En effet, Ferkessédougou est situé dans le domaine soudanais tandis que les localités de Sirasso, Nafoun et Boundiali, appartiennent au secteur sub-soudanais (Guillaumet et al., 1971 ; Beaudou et al., 1980 ; Ahoussou et al., 1995 ; Koné et al., 2002).

Le présent travail vise la caractérisation biochimique de la pulpe de prune noire issue de ces domaines en vue de fournir des informations sur la composition du fruit et l'étude de sa variabilité naturelle en fonction de ces zones climatiques. 


\section{Matériel et méthodes \\ Préparation des échantillons}

Le matériel végétal (figure 1) est constitué des prunes noires (fruit du Vitex doniana) récoltées à maturité au pied de plants sauvages pendant le mois d'Août 2016. Le mûrissement s'est fait de façon naturelle dans des paniers protégés des insectes avec du tissu en coton durant cinq jours. Les lots de fruits mûrs ont été lavés à l'eau potable et désinfectés dans un bain d'eau additionnée d'hypochlorite de sodium (200 ppm) durant 3 minutes. Ils ont par la suite été triés et stockés selon leur provenance dans une chambre froide à $-18^{\circ} \mathrm{C}$ jusqu'au moment des essais (après une semaine à un (1) mois).

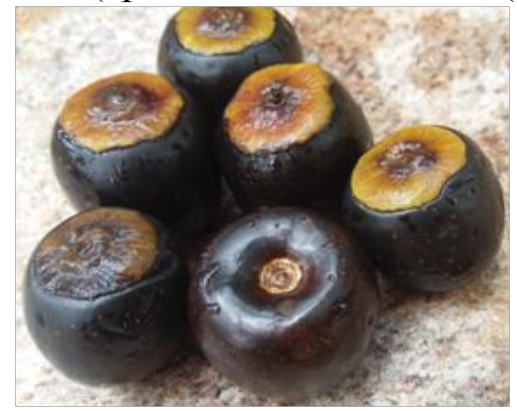

Figure 1 : Prune noire ou fruit du prunier des savanes ou fruit de Vitex doniana mûre.

\section{Extraction de la pulpe}

Les échantillons ont été sortis la veille de la chambre froide à $-18^{\circ} \mathrm{C}$ et mis à dégivrer à température ambiante Un malaxage manuel a permis la séparation de la pulpe d'avec le noyau. La pulpe a ensuite été broyée avec un mixeur plongeant (Taurus robot 300 - 300W, Espagne) durant 3 minutes. Elle a ensuite été conditionnée dans des bocaux en plastique de 0,5 L et entreposée en chambre froide pour les analyses des différents paramètres. Les pulpes ainsi conservées sont ressorties de la chambre froide 30 minutes avant chaque manipulation pour une décongélation à température ambiante.

\section{Analyse biochimique}

\section{Détermination de la teneur en eau}

Selon la méthode AOAC, (1984), des prises d'essai de $5 \mathrm{~g} \mathrm{(Me)} \mathrm{de}$ pulpe ont été placées à l'étuve (MEMERT, Schwabach West Germany) à $105^{\circ} \mathrm{C}$ pendant 24 heures. A sa sortie, la masse sèche $(\mathrm{Ms})$ a été pesée à l'aide d'une balance de précision (Sartorius BP 110 S, Germany). Les essais ont été répétés trois fois. La teneur en eau base humide (TEh) est alors calculée selon l'équation 1 :

$$
\mathrm{TE}_{\mathrm{h}}(\%)=\frac{\mathrm{Me}-\mathrm{Ms}}{\mathrm{Me}} \times 100
$$




\section{Détermination du pH}

Pour la détermination du $\mathrm{pH}$, la méthode potentiométrique de l'AOAC (1990) a été utilisée. $50 \mathrm{~mL}$ d'eau distillée ont été ajoutés à $5 \mathrm{~g}$ de pulpes ; après 10 min d'homogénéisation à l'aide d'un agitateur magnétique, le $\mathrm{pH}$ de la solution a été déterminé en utilisant un pH-mètre (HANNA HI 8424) préalablement étalonné. Les essais ont été répétés trois fois pour chacun des lots d'échantillons des 3 régions.

\section{Dosage de l'acidité titrable}

L'acidité titrable (en $\mathrm{mEq} / 100 \mathrm{~g}$ ), a été déterminée suivant la méthode colorimétrique décrite par la norme française NF V05-101 (AFNOR, 1974). On ajoute $50 \mathrm{~mL}$ d'eau distillée à une masse $(\mathrm{m})$ de $5 \mathrm{~g}$ de pulpe. Après $5 \mathrm{~min}$ d'homogénéisation à l'aide d'un agitateur magnétique et $20 \mathrm{~min}$ de centrifugation (centrifugeuse TDL-4, Chine) à $4000 \mathrm{G}$, on prélève $10 \mathrm{~mL}$ du surnageant $\left(\mathrm{V}_{0}\right)$ de normalité $\mathrm{N}_{0}$ que l'on verse dans un erlenmeyer. La titration est ensuite réalisée avec de l'hydroxyde de sodium $\left(\mathrm{NaOH}\right.$ à $\mathrm{N}_{1}=0,1$ $\mathrm{N})$ après ajout de 2 gouttes de phénolphtaléine. Soit $\mathrm{V}_{1}$, le volume de la solution de $\mathrm{NaOH}$ versé à l'équivalence. Les essais de dosage ont été répétés trois fois pour chaque lot. L'acidité titrable $\left(\mathrm{A}^{\circ}\right)$ est obtenue selon la formule de l'équation 2 :

$$
\mathrm{A}^{\circ}(\mathrm{mEq} / 100 \mathrm{~g})=\frac{\mathrm{N}_{1} \cdot \mathrm{V}_{1} \cdot 10^{5}}{\mathrm{mV}_{0}}
$$

\section{Détermination de l'extrait sec soluble}

L'extrait sec soluble (ESS) a été mesuré (en ${ }^{\circ}$ Brix) à l'aide d'un réfractomètre numérique à main (ATAGO pocket PAL- $\alpha$, Japon). Après étalonnage du réfractomètre avec de l'eau distillée, la pulpe du fruit a été pressée et quelques gouttes du jus obtenu ont été déposées sur sa lentille, suivie de la lecture du degré Brix au bout de cinq (5) secondes. Les essais ont été répétés trois (3) fois pour chaque lot.

\section{Détermination de la teneur en sucres totaux}

Les sucres totaux (en mg. $\mathrm{g}^{-1}$ ) ont été dosés en adaptant la méthode de Dubois et al. (1956). A cinq (5) g de pulpe, on ajoute $50 \mathrm{~mL}$ d'eau distillée. Après cinq (5) min d'homogénéisation à l'aide d'un agitateur magnétique et 20 min de centrifugation à $4000 \mathrm{G}$ (centrifugeuse TDL-4, Chine), $50 \mathrm{~mL} \mathrm{du}$ surnageant ont été prélevés à l'aide d'une pipette, versés dans une fiole jaugée de $200 \mathrm{~mL}$ et complétés jusqu'au trait de jauge. $100 \mu \mathrm{L}$ de cet extrait ont été versé dans un tube à essai. Puis $200 \mu \mathrm{L}$ de phénol à $5 \%$ et $1 \mathrm{~mL}$ d'acide sulfurique concentré $\left(\mathrm{H}_{2} \mathrm{SO}_{4} 95-98 \%\right)$ y ont été ajoutés. Le mélange obtenu a été chauffé au bain-marie à $100^{\circ} \mathrm{C}$ pendant 5 min et refroidi dans de la glace 
fondante. La lecture de la densité optique $\left(\mathrm{C}_{\text {lue }}\right)$ s'est faite à l'aide d'un spectrophotomètre UV visible (JASCO V-530) à $490 \mathrm{~nm}$. Les essais ont été répliqués 3 fois. La concentration des sucres totaux $\left(\mathrm{C}_{\mathrm{ST}}\right)$ a ainsi été évaluée suivant la l'équation 3 suivante :

$$
\mathrm{C}_{\mathrm{ST}}=\mathrm{C}_{\text {lue }} \times \text { Facteur de Dilution }
$$

\section{Détermination du taux de cendre}

Le taux de cendres a été déterminé selon la norme française NF V05113 (1972). Ainsi, 5 grammes de pulpe fraîche sont mis à l'étuve à $105^{\circ} \mathrm{C}$ pendant $24 \mathrm{~h}$. La pulpe séchée de masse Ms a ensuite été placée dans un four à moufles (HERAEUS) à $600^{\circ} \mathrm{C}$ pendant $5 \mathrm{~h}$ afin d'incinérer les échantillons secs. Les cendres obtenues de masse Mc ont permis de calculer le taux de cendres Tc selon l'équation 5. Les essais ont été répétés en triple pour chaque échantillon.

$$
\mathrm{T}_{\mathrm{c}}(\%)=\frac{\mathrm{Mc}}{\mathrm{Ms}} \times 100
$$

\section{Détermination du pouvoir antioxydant}

La détermination du pouvoir antioxydant s'est effectuée en modifiant légèrement la méthode de Muanda (2010) qui utilise le 2, 2-diphényl-1-picrylhydrazyle (DPPH). $50 \mathrm{~mL}$ d'eau distillée sont ajoutés à $5 \mathrm{~g}$ de pulpe. Après 5 min d'homogénéisation à l'aide d'un agitateur magnétique, le mélange est filtré à l'aide de coton hydrophile. A partir du filtrat obtenu et de l'eau distillée, des préparations de concentrations différentes ont été élaborés. Le tableau 1 présente les volumes de filtrat et d'eau distillée utilisés pour les préparations.

Tableau 1 : Volumes de filtrat et d'eau distillée utilisés pour chaque préparation.

\begin{tabular}{|c|c|c|c|c|c|c|c|}
\hline Désignation & \multicolumn{7}{|c|}{ Volumes prélevés en $\mathrm{mL}$} \\
\hline Filtrat & 2 & 1,5 & 1 & 0,5 & 0,2 & 0,1 & 0 \\
\hline Eau distillée & 0 & 0,5 & 1 & 1,5 & 1,8 & 1,9 & 2 \\
\hline
\end{tabular}

A $0,1 \mathrm{~mL}$ de chacune des préparations, on ajoute $1 \mathrm{~mL}$ d'une solution méthanolique de DPPH $(100 \mu \mathrm{M})$. L'élaboration des échantillons et du témoin se réalise suivant les mêmes procédés. Le mélange obtenu est gardé à l'abri de la lumière à température ambiante durant 20 minutes. L'absorbance a ensuite été mesurée au spectrophotomètre UV visible (JASCO V-530) à $\lambda=517 \mathrm{~nm}$ contre la solution témoin composée de $1 \mathrm{~mL}$ de la solution de DPPH et de 0,1 $\mathrm{mL}$ de méthanol. Le pourcentage d'inhibition (IP (\%)) est ensuite calculé suivant la l'équation 5:

$$
\mathrm{IP}(\%)=\frac{(\mathrm{Ao}-\mathrm{Ae})}{\mathrm{Ao}} \times 100
$$

Avec Ao : absorbance du témoin et Ae : absorbance des extraits. 
La régression linéaire établie entre les différents pourcentages d'inhibition et les concentrations permet alors de déterminer la concentration inhibitrice médiane (IC50).

\section{Dosage des polyphénols totaux}

Le dosage des polyphénols totaux (en mg Eq A.G / g matière fraîche) a été effectué par adaptation de la méthode de Wood et al. (2002). A cinq (5) $\mathrm{g}$ de pulpe, sont ajoutée $50 \mathrm{~mL}$ d'eau distillée. Après 5 min d'homogénéisation à l'aide d'un agitateur magnétique, la solution est filtrée à l'aide de coton hydrophile. Avec une pipette, $0,5 \mathrm{~mL}$ du filtrat est prélevé et renversé dans un bécher. On y ajoute $2,5 \mathrm{~mL}$ de réactif de Folin-Ciocalteu à $10 \%$. Le mélange obtenu est incubé pendant 2 min à température ambiante, à l'abri de la lumière. Ensuite, $2 \mathrm{~mL}$ de solution de carbonate de sodium $\left(\mathrm{Na}_{2} \mathrm{CO}_{3}\right)$ à 75 g.L-1 y sont ajoutés et le mélange est placé pendant $15 \mathrm{~min}$ au bain-marie à $50^{\circ} \mathrm{C}$. Après un refroidissement rapide du mélange dans de l'eau contenant de la glace, on mesure l'absorbance $(\mathrm{Cp})$ à $760 \mathrm{~nm}$ à l'aide d'un spectrophotomètre UV visible (JASCO V-530) en utilisant l'eau distillée comme blanc. Les essais ont été répétés trois (3) fois pour chaque échantillon. La teneur en polyphénols totaux $\left(\mathrm{P}_{\mathrm{t}}\right)$ est alors évaluée selon l'expression de l'équation 6 :

$$
\mathrm{P}_{\mathrm{t}}=\mathrm{C}_{\mathrm{p}} \times \mathrm{FD}
$$

\section{Dosage des flavonoïdes totaux}

Les flavonoïdes totaux (en mg QE) ont été dosés en utilisant la méthode de Marinova et $a l$. (2005). On ajoute $50 \mathrm{~mL}$ d'eau distillée à cinq (5) g de pulpe. Après 5 min d'homogénéisation à l'aide d'un agitateur magnétique, la solution est filtrée à l'aide de coton hydrophile. $2,5 \mathrm{~mL}$ de ce filtrat sont renversés dans une fiole de $25 \mathrm{~mL}$ et on y ajoute $0,75 \mathrm{~mL}$ de nitrite de sodium (NaNO2) à $5 \%$. On incorpore au mélange $0,75 \mathrm{~mL}$ de chlorure d'aluminium (AlCl3) à $10 \%$. La solution obtenue est maintenue à l'abri de la lumière pendant 6 minutes. Après incubation, $5 \mathrm{~mL}$ de soude $(\mathrm{NaOH} ; 1 \mathrm{~N})$ y sont ajoutés. Le volume est complété à $25 \mathrm{~mL}$ avec de l'eau distillée. Après une agitation vigoureuse du mélange, l'absorbance (Cf) a été mesurée au spectrophotomètre UV visible (JASCO V-530) à $\lambda=510 \mathrm{~nm}$. Pour chaque échantillon, les essais ont été répétés trois (3) fois. La proportion des flavonoïdes totaux (Cflav) est alors évaluée par l'expression de l'équation 7 :

$$
\mathrm{C}_{\mathrm{flav}}=\mathrm{C}_{\mathrm{f}} \times \mathrm{FD}
$$

\section{Détermination du taux de vitamine $\mathrm{C}$}

La concentration de vitamine C (en mg / 100g) est déterminée selon la méthode de Tillmanns et al. (1932). A cinq (5) g de pulpe sont ajoutés $50 \mathrm{~mL}$ d'eau distillée. Après $5 \mathrm{~min}$ d'homogénéisation à l'aide d'un agitateur magnétique et 20 min de centrifugation à 4000 G (centrifugeuse TDL-4, 
Chine), on recueille le surnageant dans un bécher. A $10 \mathrm{~mL}$ du surnageant, on ajoute $10 \mathrm{~mL}$ d'acide métaphosphorique pour stabiliser la vitamine C. L'échantillon à analyser est obtenu en prélevant $5 \mathrm{~mL}$ de la solution stabilisée que l'on dose ensuite dans un erlenmeyer avec un volume $(\mathrm{Ve})$ d'une solution de 2,6-dichlorophénolindophenol (2,6-DCPIP).

L'étalonnage de la solution 2,6-DCPIP a préalablement été fait avec un volume (Vs) d'acide ascorbique pur. Une autre solution préparée à partir d'acide métaphosphorique / acide acétique a également été titrée avec un volume (V0) de la solution 2,6-DCPIP. Les essais ont été répétés trois (3) fois pour tous les échantillons. La concentration en vitamine C ([vit C]) est évaluée par l'expression de l'équation 8 :

$$
[\text { Vit.C }]=\frac{2(\mathrm{Ve}-\mathrm{Vo})}{(\mathrm{Vs}-\mathrm{Vo})} \times 100
$$

\section{Détermination du taux des éléments minéraux}

Les éléments minéraux ont été quantifiés selon le protocole décrit par l'AOAC (1990). Cinq (5) g de pulpe sont disposés dans des capsules et mis à l'étuve à $105^{\circ} \mathrm{C}$ pendant $24 \mathrm{~h}$. Après refroidissement dans un dessiccateur pendant 2 heures, on prélève $0,3 \mathrm{~g}$ d'échantillon sec que l'on met dans une terrine en porcelaine qui est par la suite enfournée à $600^{\circ} \mathrm{C}$ pendant $5 \mathrm{~h}$ dans un four à moufles (HERAEUS) jusqu'à obtention d'une cendre blanche. Après 8 heures de refroidissement à température ambiante, on y ajoute $5 \mathrm{~mL}$ d'acide nitrique $\left(\mathrm{HNO}_{3}\right.$ à $\left.1 \mathrm{~N}\right)$ que l'on fait alors évaporer à sec sur une plaque chauffante. On ajoute ensuite $5 \mathrm{~mL}$ d'acide chlorhydrique $(\mathrm{HCl}, 1 \mathrm{~N})$ au résidu obtenu, et on l'enfourne à $400^{\circ} \mathrm{C}$ pendant $30 \mathrm{~min}$. Le résidu est alors récupéré dans $10 \mathrm{~mL}$ d'acide chlorhydrique $(\mathrm{HCl}, 0,1 \mathrm{~N})$ puis mis dans une fiole jaugée de $50 \mathrm{~mL}$ et on y adjoint $5 \mathrm{~mL}$ de chlorure de lantane afin d'éviter les interférences des éléments $\mathrm{Ca}$ et $\mathrm{K}$. Les éléments minéraux contenus dans la solution sont par la suite dosés par l'AAS. Les essais ont été répétés trois (3) fois pour chaque échantillon. La teneur des différents minéraux $\mathrm{T}$ (en $\mathrm{mg} . \mathrm{kg}$ $\left.{ }^{1}\right)$ est obtenue selon l'équation 9 qui suit :

$$
\mathrm{T}\left(\mathrm{mg} \cdot \mathrm{kg}^{-1}\right)=\frac{\left(\mathrm{C}_{\text {ech }}{ }^{-\mathrm{C}_{\text {blanc }}}\right) \times \mathrm{V}}{\mathrm{m}}
$$

Avec $\mathrm{C}_{\text {ech }}$ la concentration de l'échantillon lue par la machine, $\mathrm{C}_{\text {blanc }}$ la concentration du blanc donné par la machine, $\mathrm{V}$ le volume en $\mathrm{mL}$ d'acide chlorhydrique à $0,1 \mathrm{~N}$ et $\mathrm{m}$ la masse en $\mathrm{mg}$ de l'échantillon sec calciné.

\section{Analyse statistique}

Les moyennes des valeurs des différents paramètres ont été soumises à une analyse de la variance ANOVA. Le test de comparaison multiple de Welch 
a été utilisé pour déterminer les échantillons qui diffèrent les uns des autres. Il a été réalisé avec le logiciel $\mathrm{R}$ version 3.4.1. Lorsque la $\mathrm{P}$-valeur $<0,05$ (au seuil de 5\%) alors les valeurs sont significativement différentes. A contrario, $(\mathrm{P}$-valeur $>0,05$ au seuil de 5\%) les valeurs sont significativement identiques.

\section{Résultats et discussion}

La composition biochimique issue de l'analyse des pulpes de prunes noires est mise en exergue dans les tableaux 2, 3, 4, 5 et 6 qui suivent :

Teneur en eau et taux de cendre des pulpes

La figure 2 indique les différents paramètres caractérisant l'humidité de la pulpe de prune noire.

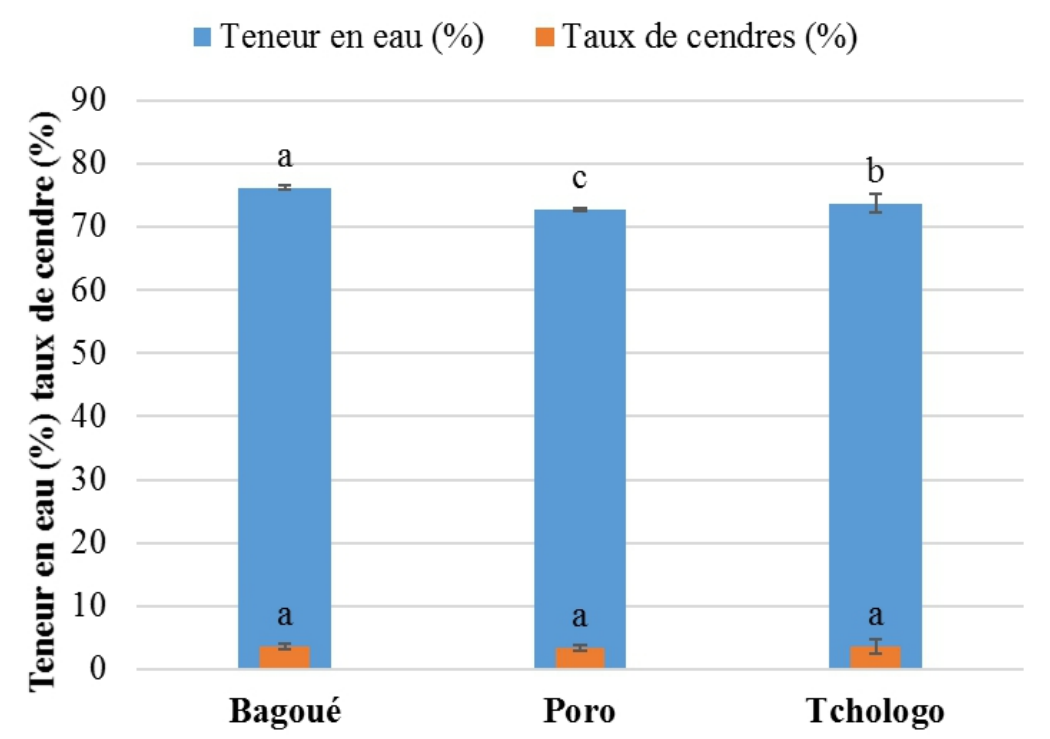

Figure 2 : Teneur en eau et taux de cendre des prunes noires dans les 3 régions de l'étude (Bagoué, Poro, Tchologo).

Pour une même couleur de la barre, lorsque les lettres au-dessus sont identiques, les moyennes sont identiques (au seuil de 5\%). Sinon, les moyennes sont significativement différentes (au seuil de 5\%).

Comme pour la plupart des fruits charnus (Ekué et al., 2008 ; Balla et $a l ., 2008)$, la teneur en eau est assez élevée et peut atteindre en moyenne 76,19 $\pm 0,29 \%$ pour la prune noire de la Bagoué. Ensuite, arrive celle du Tchologo avec 73,65 $\pm 1,46 \%$ d'eau et enfin la pulpe du Poro avec 72,69 $\pm 0,29 \%$ d'eau. Ces teneurs en eau, significativement différentes ( $\mathrm{p}$-valeur $<0,05$ ), sont quelque peu supérieures à celle trouvée par Herzog (1992) pour la prune noire dans le sud du V-Baoulé (centre de la Côte d'Ivoire). Cette forte activité en eau constitue une limite pour le temps d'entreposage de ces fruits; ce qui en fait des denrées très périssables. 
La variation du taux de cendres des pulpes n'est pas significative ( $\mathrm{p}$ valeur $>0,05)$ d'une région à l'autre comme a pu le constater Makalao et al. (2015) pour Vitex doniana et d'autres fruits au Tchad. Ces moyennes évoluant entre $3,43 \pm 0,43 \%$ et 3,64 $\pm 0,48 \%$, sont fonction de la teneur en eau et sont supérieures à la valeur 1,5\% déterminée par Herzog (1992) et inférieures à celles de Agbede et al. (2007) qui est de 5,27 $\pm 0,1 \%$. Ces proportions font de ces fruits une source non négligeable de minéraux, importants pour la nutrition humaine.

\section{Composition en acidité des pulpes}

La composition en acide des pulpes de prunes noires est présentée à la figure 3 .

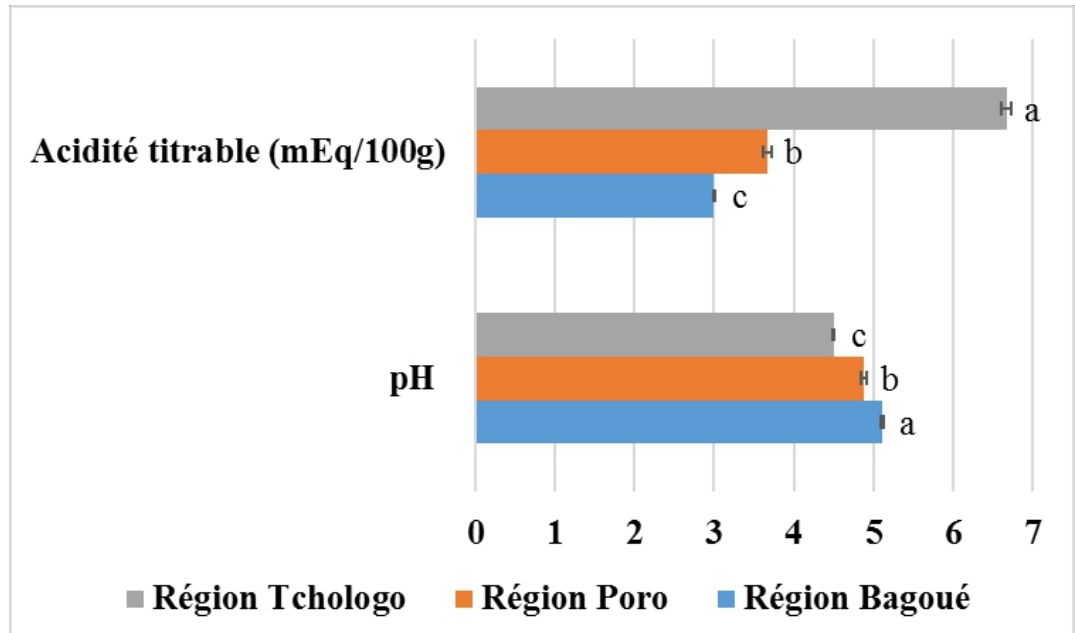

Figure 3 : Acidité de la pulpe des prunes noires dans les 3 régions d'étude (Bagoué, Poro, Tchologo).

Pour une même couleur de la barre, lorsque les lettres au-dessus sont identiques, les moyennes sont identiques (au seuil de 5\%). Sinon, les moyennes sont significativement différentes (au seuil de 5\%).

A l'analyse de la figure 3 , toutes les pulpes ont un $\mathrm{pH}$ acide qui varie entre 4,50 $\pm 0,01$ et 5,11 $\pm 0,01$. Ce qui est en concordance avec les résultats de Leung et al. (1968) et quelque peu supérieur à ceux de So et al. (2011) qui ont travaillé sur la prune noire. Ainsi, cette pulpe pourrait donner du jus avec une acidité favorable à sa stabilisation face à sa dégradation par les levures et les moisissures. Les pulpes de trois (3) régions étudiées ont des $\mathrm{pH}$ statistiquement différents ( $\mathrm{p}$-valeur $<0,05$ ) indiquant que celle du Tchologo ( $\mathrm{pH}=4,50 \pm 0,01)$ pourrait éventuellement présenter un jus plus stable que respectivement celles du Poro et de la Bagoué.

Il en est de même pour l'acidité titrable qui varie significativement ( $p$ valeur $<0,05)$ d'une région à l'autre. La pulpe de prune noire de la Bagoué a 
une acidité titrable la plus faible tandis que celle du Tchologo a la teneur la plus élevée.

Ces résultats montrent la corrélation entre la valeur du pH et celle de l'acidité titrable de la pulpe de prune noire. Lorsque l'acidité libre augmente, on note une diminution du $\mathrm{pH}$.

\section{Composition en sucres des pulpes}

La composition moyenne en sucres des pulpes du fruit de Vitex doniana, représentée par l'extrait sec soluble et les sucres totaux, est présentée à la figure 4 .

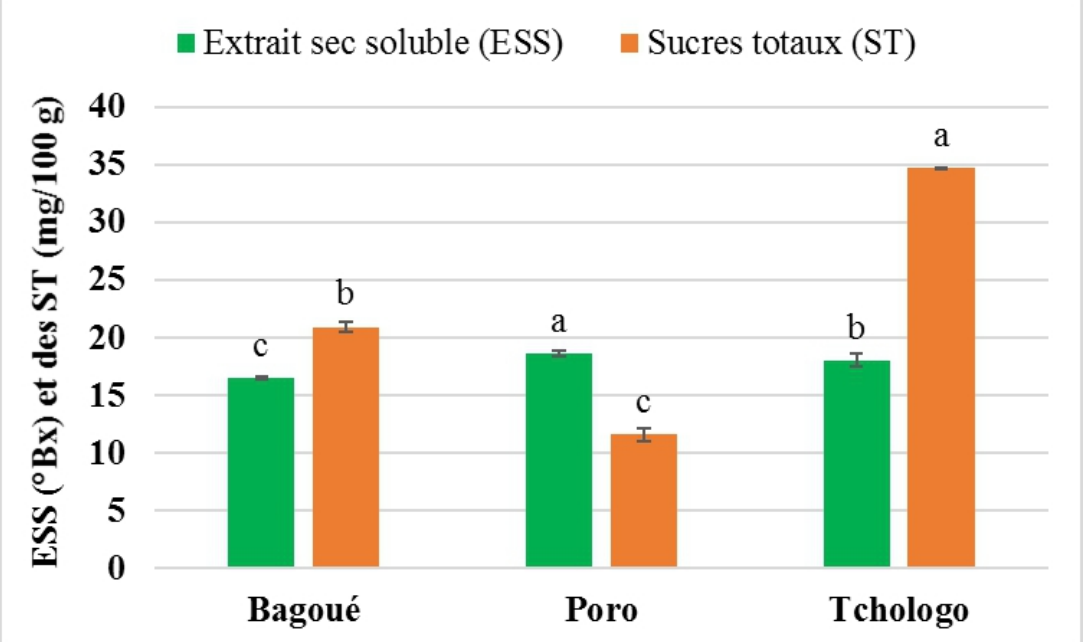

Figure 4 : Composition en sucres des pulpes du fruit de Vitex doniana dans les 3 régions de l'étude (Bagoué, Poro, Tchologo).

Pour une même couleur de la barre, lorsque les lettres au-dessus sont identiques, les moyennes sont identiques (au seuil de 5\%). Sinon, les moyennes sont significativement différentes (au seuil de 5\%).

Pour l'extrait sec soluble des pulpes, les moyennes sont significativement différentes (p-valeur $<0,05)$ d'une région à l'autre. Ces valeurs sont de $16,53 \pm 0,12{ }^{\circ} \mathrm{B}, 18,57 \pm 0,25{ }^{\circ} \mathrm{B}$ et $18,03 \pm 0,55{ }^{\circ} \mathrm{B}$ respectivement pour les régions de la Bagoué, du Poro et du Tchologo. So et al. (2011) ont trouvé une valeur de $18.83 \pm 0.11^{\circ} \mathrm{B}$ pour la pulpe de prune noire lors de leurs travaux. Ces valeurs comparables avec celles trouvées par Dick et al. (2009) pour la mangue Kent du nord de la Côte d'Ivoire, indiquent que la prune noire est très riche en sucres ; ce qui reste un critère de choix dans l'appréciation du goût sucré des fruits mais représente également un risque de dégradation par les levures en cas de mauvaise conservation.

Pour les sucres totaux (ST), les valeurs moyennes comprises entre $11,60 \pm 0,60 \mathrm{mg} 100 \mathrm{~g}$ et $34,67 \pm 0,08 \mathrm{mg} / 100 \mathrm{~g}$; on note aussi une variation significative (p-valeur $<0,05)$ du taux de sucres d'une région à l'autre. Ces 
valeurs traduisent la proportion de sucres réducteurs dans la pulpe de prune noire. La pulpe des fruits provenant de la région du Tchologo sont les plus riches en ST, suivie de celles de la Bagoué et celle du Poro qui enregistre la teneur en ST la plus basse. Cette concentration en ST de la pulpe de ce fruit du nord de la Côte d'Ivoire, est plus élevée que celle de la pulpe des fruits du Baobab (Adansonia digitata) (Cissé et al., 2009) et du jujubier (Zizyphus mauritiana Lam.) (Danthu et al., 2002) au Sénégal.

\section{Composition en antioxydants des pulpes}

Le tableau 2 résume les différentes teneurs en antioxydants de la pulpe de prune noire.

Tableau 2 : Proportion antioxydante de la pulpe des prunes noires dans les 3 régions de l'étude (Bagoué, Poro, Tchologo)

\begin{tabular}{|c|c|c|c|}
\hline \multirow{2}{*}{ Paramètres } & \multicolumn{3}{|c|}{ Régions } \\
\hline & Bagoué & Poro & Tchologo \\
\hline $\begin{array}{l}\text { Pouvoir antioxydant } \\
\left(\text { IC50 }\left(\mu \mathrm{g} . \mathrm{mL}^{-1}\right)\right)\end{array}$ & $18,63 \pm 0,02^{\mathrm{a}}$ & $27,80 \pm 0,01^{b}$ & $35,54 \pm 0,01^{\mathrm{c}}$ \\
\hline $\begin{array}{l}\text { Polyphénols totaux (mg } \\
\left.\text { EqA.G. } 100 \mathrm{~g}^{-1}\right)\end{array}$ & $193,33 \pm 0,03^{a}$ & $196,67 \pm 0,01^{b}$ & $166,67 \pm 0,01^{\mathrm{c}}$ \\
\hline $\begin{array}{l}\text { Flavonoïdes totaux (mg } \\
\left.\text { Q.E. } 100 \mathrm{~g}^{-1}\right)\end{array}$ & $138,67 \pm 2,31^{\mathrm{a}}$ & $106,67 \pm 2,31^{b}$ & $157,33 \pm 0,58^{\mathrm{c}}$ \\
\hline Vitamine $\mathrm{C}\left(\mathrm{mg} .100 \mathrm{~g}^{-1}\right)$ & $15,05 \pm 0,07^{\mathrm{a}}$ & $14,70 \pm 0,06^{\mathrm{a}}$ & $14,34 \pm 0,02^{a}$ \\
\hline
\end{tabular}

Lorsque pour une même ligne, les lettres en exposant sont identiques, les moyennes sont identiques au seuil de 5\%. Sinon, les moyennes sont significativement différentes au seuil de $5 \%$.

- L'étude du pouvoir antioxydant des pulpes de fruits des trois (3) régions montre des valeurs de concentrations inhibitrices médianes (IC50) qui varient entre $18,63 \mathrm{mg} / \mathrm{ml}$ et $35,54 \mathrm{mg} / \mathrm{ml}$. L'IC50 est inversement lié à la capacité antioxydante d'un composé, car il exprime la quantité d'antioxydant requise pour diminuer la concentration du radical libre de $50 \%$. Plus la valeur d'IC50 est basse, plus l'activité antioxydante d'un composé est grande (Barkat et Laib, 2011). Ce qui indique que la pulpe de fruits en provenance de la Bagoué présente l'activité antioxydante la plus élevée $(18,63 \pm 0,02 \mu \mathrm{g} / \mathrm{ml})$. Vient ensuite celle du Poro $(27,80 \pm 0,01 \mu \mathrm{g} / \mathrm{ml})$ et enfin celle du Tchologo qui a la capacité antioxydante la plus faible $(35,54 \pm 0,01 \mu \mathrm{g} / \mathrm{ml})$. L'analyse statistique de ces différentes valeurs indique qu'elles sont significativement différentes au seuil de 5\%.

La teneur en polyphénols totaux est de 193,33 $\pm 0,03 \mathrm{mg}$ Eq A.G/100g pour la pulpe de prune noire de la Bagoué, 196,67 \pm 0,01 mg Eq A.G/100g pour le Poro et $166,67 \pm 0,01 \mathrm{mg}$ Eq A.G/100g pour le Tchologo. L'analyse statistique montre que ces valeurs sont significativement différentes. Une consommation régulière de la pulpe de prune noire pourrait permettre d'éviter les troubles liés au stress oxydatif comme les maladies dégénératives ( $\mathrm{Li}$ et $a l$., 
2007). Les moyennes de flavonoïdes totaux sont statistiquement différentes pour toutes les régions étudiées avec 138,67 $\pm 2,31 \mathrm{mg}$ Q.E/100g pour la Bagoué, 106,67 $\pm 2,31 \mathrm{mg}$ Q.E/100g pour le Poro et 157,33 $\pm 0,58 \mathrm{mg}$ Q.E/100g pour le Tchologo. Cette concentration est un atout pour la santé puisque les flavonoïdes de par leur fonction, protègent les vaisseaux sanguins des dommages liés au cholestérol (Abdou, 2009).

La pulpe du fruit est riche en vitamine C. Les moyennes obtenues sont dans l'ordre de 14,34 $\pm 0,02 \mathrm{mg} / 100 \mathrm{~g}$ à 15,05 $\pm 0,07 \mathrm{mg} / 100 \mathrm{~g}$. Ces valeurs n'ont pas montré de différences statistiquement significatives pour toutes les régions et se situent dans l'intervalle $6-18 \mathrm{mg} / 100 \mathrm{~g}$ trouvé par Leung et al. (1968). Elles sont par contre inférieures à la valeur déterminée par So et al. (2011). Ce fruit présente une teneur en vitamine $C$ bien supérieure à celle d'un autre fruit sauvage, Dialium guineense du Bénin, étudié par Gnansounou et al. (2014). La consommation de la prune noire pourrait être bénéfique pour la santé vu le rôle protecteur et le pouvoir antioxydant de la vitamine $\mathrm{C}$ (FAO/WHO, 2002).

A l'analyse des résultats, les antioxydants que sont les polyphénols et la vitamine $\mathrm{C}$ sont en quantité plus importante dans la pulpe de la prune noire, provenant de la Bagoué. Cela expliqueraient la forte activité antioxydante de la pulpe de prune noire de cette région. Toutefois, celle du Tchologo, malgré sa quantité moyenne en antioxydants totaux, a une activité antioxydante plus faible, comparée à celle du Poro (qui a la quantité totale en antioxydants la plus faible).

\section{Composition en minéraux des pulpes}

La composition minérale de la pulpe de prune noire pour les 3 régions de l'étude est présentée dans le tableau 3.

Tableau 3 : Teneur en minéraux de la pulpe de prune noire pour les 3 régions de l'étude.

\begin{tabular}{|c|c|c|c|}
\hline \multirow{2}{*}{ Paramètres } & \multicolumn{3}{|c|}{ Régions } \\
\hline & Bagoué & Poro & Tchologo \\
\hline Mg (mg. 100 g-1) & $4,11 \pm 2,08^{a}$ & $2,16 \pm 0,73^{c}$ & $2,36 \pm 0,27^{b}$ \\
\hline K (mg. 100 g-1) & $53,68 \pm 6,18{ }^{a}$ & $38,00 \pm 9,46^{b}$ & $36,67 \pm 8,88^{c}$ \\
\hline Ca (mg. $100 \mathrm{~g}-1)$ & $19,57 \pm 5,93^{b}$ & $21,89 \pm 1,13^{a}$ & $14,15 \pm 1,00^{c}$ \\
\hline
\end{tabular}

Lorsque pour une même ligne, les lettres en exposant sont identiques, les moyennes sont identiques au seuil de 5\%. Sinon, les moyennes sont significativement différentes au seuil de $5 \%$.

Après les différentes analyses minérales, les moyennes obtenues sont toutes significativement différentes pour tous les minéraux.

Les teneurs en calcium sont de 19,57 $\pm 5,93 \mathrm{mg} / 100 \mathrm{~g}$ pour la Bagoué, $21,89 \pm 1,13 \mathrm{mg} / 100 \mathrm{~g}$ pour le Poro et $14,15 \pm 1,00 \mathrm{mg} / 100 \mathrm{~g}$ pour le Tchologo. Le fruit du Tchologo est plus riche en calcium que la pulpe du fruit du pommier 
de Cayor (Neocarya macrophylla Sabine) étudiée au Bénin par Balla et al. (2008).

Pour ce qui est de la teneur des pulpes en potassium, les moyennes sont de 53,68 $\pm 6,18 \mathrm{mg} / 100 \mathrm{~g}$ pour la Bagoué, 38,00 \pm 9,46 mg/100g pour le Poro et $36,67 \pm 8,88 \mathrm{mg} / 100 \mathrm{~g}$ pour le Tchologo. C'est le minéral le plus abondant tout comme dans la pulpe de baobab et la plupart des végétaux (Cissé, 2012). La pulpe de la prune noire est donc une source de potassium non négligeable. La consommation de ce fruit pourrait aider à combler le besoin nutritionnel journalier en potassium et à éviter des disfonctionnements au niveau de l'organisme humain.

La teneur en magnésium de la pulpe de prune noire de la Bagoué est de 4,11 $\pm 2,08 \mathrm{mg} / 100 \mathrm{~g}$, celle du Poro de 2,16 $\pm 0,73 \mathrm{mg} / 100 \mathrm{~g}$ et celle du Tchologo de 2,36 $\pm 0,27 \mathrm{mg} / 100 \mathrm{~g}$. Elles contiennent moins de magnésium que les prunes noires du Tchad étudiées par Makalao et al. (2015).

Quel que soit l'élément minéral dosé (calcium, potassium ou magnésium), les moyennes ont montré des variations significatives d'une région à l'autre. Des études réalisées par Herzog (1992) avaient déterminées des valeurs de $18,1 \mathrm{mg} / 100 \mathrm{~g}$ pour le calcium, $15,53 \mathrm{mg} / 100 \mathrm{~g}$ pour le magnésium et $679 \mathrm{mg} / 100 \mathrm{~g}$ pour le potassium. So et $\mathrm{al}$. (2011) ont déterminés des teneurs de $0,765 \mathrm{mg} / 100 \mathrm{~g}$ pour le calcium et $1,33 \mathrm{mg} / 100 \mathrm{~g}$ pour le magnésium. La consommation de la pulpe de ce fruit pourrait donc participer à la satisfaction du besoin de l'organisme humain en termes de minéraux et contribuer ainsi à une bonne croissance, au bon déroulement du processus métabolique et à la prévention de certaines maladies (FAO/WHO, 2002).

\section{Conclusion}

Cette étude a permis d'une part de déterminer la composition biochimique et le potentiel nutritionnel des pulpes de la prune noire de 3 régions (Bagoué, Poro et Tchologo) du nord de la Côte d'Ivoire. D'autre part, elle a permis d'établir la variabilité de cette composition d'une région à l'autre.

La pulpe de prune noire se caractérise par son acidité, une richesse en eau, en sucre et en éléments minéraux. Elle contient également une teneur élevée en vitamine $\mathrm{C}$ et en composés phénoliques dont les flavonoïdes. Le pouvoir antioxydant élevé de la pulpe pourrait avoir une liaison directe avec la présence de ces substances antioxydantes.

La variation de composition des pulpes de fruits selon la zone de récolte pourrait s'expliquer par l'influence de certains facteurs tels que la physiologie et la génétique des plants, le degré de maturation des fruits, le type de sol ou encore les conditions environnementales (température, rayonnement, pluviométrie) propres à chacune des régions (Bergeron, 1995 ; Bautista et al., 2007 ; Causse et al., 2007 ; Génard et al., 2010 ; Mauget et al., 2011). 
En termes d'apport nutritionnel, la richesse de la prune noire en antioxydants et minéraux est une source intéressante exploitable pour l'atteinte d'un régime alimentaire satisfaisant et équilibré. De même, la mise en place de techniques de valorisation de sa pulpe permettront d'atteindre un plus large public et rendront cette ressource disponible hors saison. La variabilité de la composition biochimique pourrait permettre en fonction de la qualité de produit final recherchée, d'utiliser les fruits de différentes régions sans toutefois avoir recours à des composés chimiques pas toujours inoffensifs pour la santé.

\section{References:}

1. Abdou, B.A., 2009. Contribution à l'étude du développement d'un aliment fonctionnel à base d'épices du Cameroun: caractérisation physico-chimique et fonctionnelle. Institut National Polytechnique de Lorraine, Université De Ngaoundere, Ensai, 228 p.

2. Agbede J. O., Ibitoye A. A., 2007. Chemical composition of black plum (Vitex doniana): an under-utilized fruit. Journal of Food, Agriculture \& Environment Vol.5 (2), 95-96.

3. Ahoussou, N., Koffi, G., Sangaré, A., Sangaré, A., 1995. Côte d'Ivoire : rapport de pays pour la conférence technique internationale de la FAO sur les ressources phytogénétiques. Fao, Leipzig, Allemagne, 75 p.

4. AISA, 1991. Le développement agro-pastoral et agroindustriel du nord de la Côte-d'Ivoire: cas des départements de Korhogo, Boundiali, Ferkessédougou. AISA, Abidjan, 133 p.

5. Ambé, G., 2001. Les fruits sauvages comestibles des savanes guinéennes de Côte d'Ivoire: état de la connaissance par une population locale, les Malinké. Biotechnol. Agron. Soc. Environ., 5(1), 43-58.

6. Arbonnier, M., 2000. Arbres, arbustes et lianes des zones sèches d'Afrique de l'Ouest. CIRAD-MNHN-UICN, France, 541 p.

7. AFNOR, 1974. Produits dérivés des fruits et légumes - Détermination de l'acidité titrable. Association Française de Normalisation, Paris, NF V 05-101, 1-4.

8. AOAC, 1984. Official methods of analysis, Association of official Analytical Chemists: 7.003. 14th edition, Washington DS.

9. AOAC, 1990. Official methods of analysis of the association of official analytical chemists: 981.12 Arlington, USA, 15(2), 910-928.

10. Balla, A., Barage, M., 2008. Analyses physico-chimiques de la pulpe et caractérisation de la fraction lipidique des amandes du fruit du pommier de Cayor (Neocarya macrophylla sabine). Bull. Rech. Agron. Bénin, 6 p. 
11. Barkat, M, Laib, I., 2011. Composition chimique et activité antioxydante de l'huile essentielle des fleurs sèches de Lavandula officinalis. Revue de génie industriel, 6, 46-54.

12. Bautista, O., Fernandez, F., Lopez, R., Gomez, P., 2007. The effects of œnological practices inanthocyanins, phenolic compounds and wine colour and their dependence on grape characteristics. Journal of Food Composition and Analysis, 20, 546-552.

13. Beaudou A.G., Sayol R., 1980. Etude Pédologique De La Région De Boundiali-Korhogo (Côte d'Ivoire). O.R.S.T.O.M., Paris, France, note explicative 84, 58 p. ISBN 2-7099-0554-X

14. Bergeron, L., 1995. Effet de la teneur en eau du sol sur le rendement et la qualité des fruits du bleuet nain. Mémoire de maîtrise en ressources renouvelables, Université du Québec, 81 p.

15. Causse, M., Chervin, C., Mauget, J-C., Renard, C., Fournier, D., 2007. Chapitre 2: Les sources de variabilité des qualités nutritionnelles des fruits et légumes. ESCo "Les fruits et légumes dans l'alimentation", 173-221.

16. CTA, 2007. Les fruits indigènes. Programme de radio rurale 07/5. Centre technique de coopération agricole et rurale, Wageningen, The Netherlands.

17. Cissé I., 2012. Caractérisation des propriétés biochimiques et nutritionnelles de la pulpe de baobab des espèces endémiques de Madagascar et d'Afrique continentale en vue de leur valorisation. Rapport de thèse, Génie des procédés, Montpellier SUPAGRO, 167 p.

18. Cissé, M., Sakho, M., Dornier, M., Diop, M.C., Reynes, M., Sock, O., 2009. Caractérisation du fruit du baobab et étude de sa transformation en nectar. Fruits, CIRAD/EDP Sciences 64, 19-34.

19. Dadjo, C., Assogbadjo, A.E., Fandohan, B., Glèlè Kakaï, R., Chakeredza, S., Houehanou, T.D., Damme, P.V., Sinsin, B., 2012. Uses and management of black plum (Vitex doniana Sweet) in Southern Benin. Fruits 67, 239-248.

20. Danthu, P., Soloviev, P., Totte, A., Tine, E., Ayessou, N., Gaye, A., Niang, D.T., Seck, M., Fall, M., 2002. Caractères physico-chimiques et organoleptiques comparés de jujubes sauvages et des fruits de la variété Gola introduite Au Sénégal. Fruits, CIRAD/EDP Sciences 57, 173-182.

21. Dick, E., Adopo, N.A., Camara, B., Moudioh, E., 2009. Influence of maturity stage of mango at harvest on its ripening quality. Fruits, EDP Sciences 64, 13-18. Doi:10.1051/Fruits:2008045

22. Dubois M., Gilles K.A., Hamilton J.K., Robert P.A., Smith F., 1956. Colorimetrymethod for determination of sugar and related substances. Ann. Chem. 28, 350-356. 
23. Food and Agriculture Organization (FAO), 2008. Programme d'éducation nutritionnelle intégré aux centres nutritionnels communautaires Côte d'Ivoire. Division 'Nutrition et Protection du Consommateur' de la FAO (AGN), 2 p.

24. Food and Agriculture Organization (FAO)/World Health Organisation (WHO), 2002. Chapter 6 Vitamin C, In: human vitamin and mineral requirements. Agriculture and consumer protection, Rome, Pp. 73-87.

25. Génard, M., Robin, C., Gautier, H., Massot, C., Bénard, C., Larbat, R., Bertin, N., Le Bot, J., Adamowicz, S., Bourgaud, F., 2010. Elaboration de la qualité du fruit : composition en métabolites primaires et secondaires. Innovations Agronomiques 9, 47-57.

26. Gnansounou, S. M., Noudogbessi, J. P., Yehouenou, B., Gbaguidi, A. N. M., Dovonon, L., Aina, M. P., Ahissou, H., Sohounhloue, D., 2014. Proximate composition and micronutrient potentials of Dialium guineense wild growing in Benin. International Food Research Journal 21(4), 1603-1607.

27. Guillaumet J.-L., Adjanohoun E., 1971. Le milieu naturel de la Côte d'Ivoire : La végétation. O.R.S.T.O.M., Paris, France, 161-262.

28. Herzog, F.M., 1992. Etude biochimique et nutritionnelle des plantes alimentaires sauvages dans le Sud du V-Baoulé, Côte d'Ivoire. Ecole Polytechnique Fédérale Zürich, Suisse, 8 p.

29. Koné M. W., Atindéhou K. K., Téré H., Traoré D., 2002. Quelques plantes médicinales utilisées en pédiatrie traditionnelle dans la région de Ferkessédougou (Côte d'Ivoire). Bioterre, Rev. Inter. Sci. de la Vie et de la Terre, $\mathrm{N}^{\circ}$ spécial, Editions Universitaires de Côte d'Ivoire, 7 p.

30. Ky, K. J. M., 2008. Vitex doniana Sweet. PROTA (Plant Resources of Tropical Africa / Ressources végétales de l'Afrique tropicale), Wageningen, Netherlands.

31. Leung,W.-T.W., Busson, F., Jardin, C., 1968. Food composition table for use in Africa. Rome, FAO.

32. Li W., Gao Y., Zhao J., Wang Q., 2007. Phenolic, Flavonoid, and Lutein

Ester Content and Antioxidant Activity of 11 Cultivars of Chinese Marigold. J. Agric. Food Chem, 55, 8478-8484.

33. Makalao, M.M., Savadogo, A., Zongo, C., Traoré, S.A., 2015. Composition nutritionnelle de 10 fruits sauvages consommés dans trois départements du Tchad. Int J Biol Chem Sci 9, 2385-2400.

34. Malzy, P., 1954. Quelques plantes du Nord-Cameroun et leurs utilisations. JATBA 39, 5- 6. 
35. Marinova, D., Ribarova, F., Atanassova, M., 2005. Total phenolics and total flavonoids in bulgarian fruits and vegetables. Journal of the University of Chemical Technology and Metallurgy, 40(3), 255-260

36. Mauget, J.C., Causse, M., 2011. Qualités nutritionnelles des fruits et légumes et itinéraires techniques. Pratiques agricoles innovantes et qualités nutritionnelles des produits alimentaires. Séminaire INRADGAL, 1-16

37. Ministère de la Santé et de L'hygiène publique (MSHP) / Programme National Nutrition (PNN), 2010. Guide national de soins et soutien nutritionnels et alimentaires pour les personnes affectées et infectées par le VIH et/ou la tuberculose. $103 \mathrm{p}$.

38. Muanda, F.N., 2010. Identification de polyphénols, évaluation de leur activité antioxydante et étude de leurs propriétés biologiques. Thèse de doctorat de l'Université Paul Verlaine-Metz, 295 p.

39. NF V 05-113, 1972. Détermination de la teneur en cendres.

40. N'goran P., 2014. Sécurité Alimentaire et Nutritionnelle En Côte d'Ivoire : Etat des lieux, défis. Programme National de Nutrition, $42 \mathrm{p}$.

41. Ngwamashi, E., 2009. Inventaire des espèces ligneuses locales pour le reboisement à des fins énergetiques. Université de Kinshasa.

42. Nwachukwu, E., Uzoeto, H. O., 2010. Antimicrobial activities of leaf of Vitex doniana and Cajanus cajan on some bacteria. Researcher 2(3), $37-47$.

43. Okafor, J. C., 1991. Amélioration des essences forestières donnant des produits comestibles. UNASYLVA 165, 83-96.

44. Sanogo, R., Karadji, A. H., Dembélé, O., Diallo D., 2009. Hypertension artérielle. Med. 24 (4), 1-6.

45. Schwartz H., Grellety Y., 2010. Evaluation de la prise en charge de la malnutrition aiguë Côte d'Ivoire: Rapport de mission. UNICEF Bureau Régional de l'Afrique de l'Ouest et du Centre, $29 \mathrm{p}$.

46. So, A.-S., Jo, A., 2011. Physico-chemical properties and sensory evaluation of jam made from black-plum fruit (Vitex doniana). Afr. J. Food Agric. Nutr. Dev. 11(3), 2011 pp.

47. Stevens, G. A., Finucane M. M., De-Regil L. M., Paciorek C. J., Flaxman S. R., Branca F., Ezzati M., 2013. Global, regional, and national trends in haemoglobin concentration and prevalence of total and severe anaemia in children and pregnant and non-pregnant women for 1995-2011: a systematic analysis of population-representative data. The Lancet Global Health 1 (1), 16-25. doi: 10.1016/s2214$109 x(13) 70001$.

48. Tapé C., Samassi D., Yao N. J., Déza D. A., Ba Z. S., Yao K. E., Boti B. B. D., Touré A., Yao K. H., Gue T. A. M., Abou T. H., Goh Y. E., 
2015. Enquête sur le niveau de vie des ménages en Côte d'Ivoire. Institut National de la Statistique, $91 \mathrm{p}$.

49. Tillmans, H.P., Hirsch, W., 1932. Ztschr. Unters. Lebensmittel, 63, 121.

50. UEMOA/ PRSA, 2002. Appui à la mise en œuvre de la politique agricole de l'union en matière de sécurité alimentaire : Propositions d'investissement dans le domaine de la sécurité alimentaire. Union Economique et Monétaire Ouest Africaine / Programme Régional de Sécurité Alimentaire, $31 \mathrm{p}$.

51. Li W., Gao Y., Zhao J., Wang Q., 2007. Phenolic, Flavonoid, and Lutein

Ester Content and Antioxidant Activity of 11 Cultivars of Chinese Marigold. J. Agric.Food Chem, 55, 8478-8484.

52. OMS, 2009. Global prevalence of vitamin A deficiency in populations at risk 1995-2005. WHO global database on vitamin A Deficiency. Available from: http://whqlibdoc.who.int/publications/2009/9789241598019_eng.pdf

53. Wood, J.E., Senthilmohan, S.T., Peskin, A.V., 2002. Antioxidant activity of procyanidin-containing plant extracts at different $\mathrm{pHs}$. Food Chemistry, 77(2), 155-161. httpI//dx.doi.org/10.1016/S03088146(01)00329-6. 